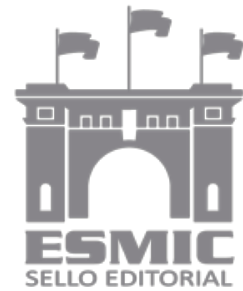

Revista Científica General José María Córdova

ISSN 1900-6586 (impreso), 2500-7645 (en línea)

Volumen 16, Número 22, abril-junio 2018, pp. 109-132

http://dx.doi.org/10.21830/19006586.324

Citación: Campion Canelas, M. (2018, abril-junio). Las fronteras como espacios de poder y resistencia en el periodo colonial. Rev. Cient. Gen. José María Córdova, 16 (22), 109-132. DOI: http://dx.doi. org/10.21830/19006586.324

\title{
Las fronteras como espacios de poder y resistencia en el periodo colonial
}

Sección: Historia

Artículo de investigación científica y tecnológica

\author{
Minerva Campion Canelas ${ }^{*}$ \\ Pontificia Universidad Javeriana, Bogotá, Colombia
}

Borders as spaces of power and resistance in the colonial period

Fronteiras como espaços de poder e resistência no período colonial

Les frontières comme espaces de pouvoir et de résistance à l'époque coloniale

Recibido: 12 de junio de 2016 • Aceptado: 14 de marzo de 2018

* https://orcid.org/0000-0003-1447-9318 - Contacto: m.campionc@javeriana.edu.co 
Resumen. Este artículo analiza a partir de una perspectiva holística, el desarrollo de las fronteras territoriales y sociales del Virreinato de Perú. La discusión se centra en la creación, avance, retroceso y transformación de las fronteras en la Audiencia de Quito. Para ello, comprendemos los procesos de expansión y retroceso de las fronteras en términos de relaciones de poder. En este sentido, se evidencia no sólo la expansión de la frontera colonial, sino también las experiencias de resistencia indígena en las regiones fronterizas.

Palabras clave: colonia; colonización; fronteras del Virreinato del Perú; historia latinoamericana; población indígena.

Abstract. This paper analyses, through a holistic approach, the development of the territorial borders and social boundaries of the Viceroyalty of Peru, focusing on the Audience of Quito. For this purpose, we understand the expansion and retreat processes of borders in terms of power relations. In this sense, we not only demonstrate the expansion of the colonial boundaries, but also the indigenous resistance experiences in the border regions.

Keywords: boundaries of the Viceroyalty of Peru; Colonial era; colonization; indigenous peoples; Latin American History.

Resumo. Este artigo analisa, a partir de uma perspectiva holística, o desenvolvimento das fronteiras territoriais e sociais do Vice-reinado do Peru. A discussão centra-se na criação, avanço, recuo e transformação das fronteiras na Audiência de Quito. Para isso, entendemos os processos de expansão e recuo das fronteiras em termos de relaçóes de poder. Nesse sentido, fica evidente não apenas a expansão da fronteira colonial, mas também as experiências de resistência indígena nas regiōes de fronteira.

Palavras-chave: A história da América Latina; colônia; colonização; fronteiras do Vicereinado do Peru; população indígena.

Résume. Cet article analyse, d'un point de vue holistique, le développement des frontières territoriales et sociales de la vice-royauté du Pérou. La discussion se concentre sur la création, l'avancement, le retrait et la transformation des frontières de l'Audiencia de Quito. Pour ce faire, nous comprenons les processus d'expansion et de retrait des frontières en termes de relations de pouvoir. En ce sens, il est évident non seulement l'expansion de la frontière coloniale, mais aussi les expériences de résistance indigène dans les régions frontalières.

Mots-clés : colonie ; colonisation ; L'histoire de l'Amérique latine ; frontières de la vice-royauté du Pérou ; population indigène. 


\section{Introducción}

En este trabajo vamos a investigar la configuración territorial de la Audiencia de Quito de acuerdo con el retroceso y avance de las fronteras que fueron establecidas con la colonización y expansión de la conquista española. Para realizar esta tarea proponemos una serie de objetivos que obedecen a la estructura del presente artículo. En primer lugar, comprenderemos la concepción de la frontera en el Estado inca o Tawantinsuyu, para después, poder analizar la alteración de las estructuras sociales y territoriales que implicó la expansión de la frontera colonial. Este análisis se realizará considerando la conquista de la Sierra, la Costa y la Amazonía. Asimismo, como entendemos que la expansión de una frontera está constituida por relaciones de poder, revisaremos los procesos de resistencia indígena que surgieron desde algunos espacios fronterizos del Virreinato de Perú. En cuarto lugar, proponemos una lectura de la frontera desde una perspectiva social y territorial, es decir, entendiendo que las instituciones coloniales utilizaron el territorio como lógica de control social y control racial. En quinto lugar, retomamos las reformas borbónicas ocurridas a principios del siglo XVIII, puesto que implicaron una nueva forma de concepción del territorio imbricado en las lógicas del Estado-nación. Por último, presentamos las conclusiones del trabajo.

Asimismo, este artículo se basa en un doble ejercicio. Por un lado, en comprender la expansión de las fronteras coloniales durante la creación del Virreinato de Perú, y por otro, en detallar algunos de los procesos de resistencia indígena que ocurrieron durante ese periodo en los espacios fronterizos ${ }^{1}$. Para ello, utilizamos el concepto de frontera también en dos sentidos, uno territorial y otro social; ambos imbricados en relaciones de poder. Es decir, este término nos permite evidenciar la existencia de espacios de tensión, de conquista y de resistencia, en las regiones fronterizas. Es por esta razón que utilizamos la concepción de frontera propuesta por O'Donnell (2002, p. 8), quien la entiende como un elemento constitutivo de las relaciones de poder, ya que su expansión implica la dominación de habitantes que "ya estaban ahî". Si bien consideramos que la revisión de la presente temática es útil para comprender tanto la formación de los Estados latinoamericanos, como el mantenimiento de las jerarquías raciales en el continente; centramos nuestro artículo en la revisión de las fronteras del Estado inca y, sobre todo, en la expansión de la frontera colonial hispánica a través de la alteración de las estructuras indígenas.

$1 \quad$ Si bien consideramos en una perspectiva global el Virreinato de Perú, centramos el estudio sobre la Audiencia de Quito. 


\section{El periodo previo a la conquista: \\ las fronteras del Tawantinsuyu}

Los pueblos indígenas de América Latina no siempre han vivido bajo la misma división política, administrativa y territorial que encontramos hoy en día. Aunque en los tiempos de antes de la conquista no existía la noción que hoy tenemos de frontera, podemos observar que para los pueblos indígenas también existían otra clase límites que "lograban diferenciar el yo del otro" (Rhi-Sausi y Oddone, 2009, p. 11). Cabe recordar también que, aunque hablamos sobre el establecimiento de fronteras, no era una "clara ni materializada noción de frontera tal como la conocemos en nuestros días" (Portais, 1983, 61). Asimismo, estas sociedades organizaban el territorio de forma horizontal, lo que significa que en vez de la fundamentación de la geometría euclidiana que representa los mapas a través de líneas imaginarias, el territorio se comprendía desde la perspectiva de las características fisiográficas (Johnson y White, 2011, p. 2141).

In examining classical Indigenous traditions, we see Indigenous peoples also held concern about boundaries and membership, but did so in ways that stressed the fundamental connectedness of human life, meeting their needs for identity, security, and order through cosmologically narrated relationships of material exchange and mobility (Costa, 2007, p. 15).

Antes de la llegada de los primeros conquistadores existieron varios lugares marcadamente diferenciados social y geográficamente en América Latina. Mientras que la Sierra condensó un elevado grado de centralización social y política, y correspondía con el Tawantinsuyu; la Amazonía se componía de diversos pueblos indígenas que vivían bajo un sistema descentralizado, pero con una gran cohesión social (Maldonado, 2006, pp. 27-30). Asimismo, la Costa estaba formada por una variedad de pueblos diferenciados entre sí (Romoli, 1963). Geográficamente, los incas poblaban la zona de los valles, aunque llegaron a asimilar y conquistar otros pueblos del altiplano. La región amazónica, en cambio, no pudo ser conquistada (Casanova-Velázquez 2000, p. 140).

Poco antes de la llegada de los espańoles a la costa ecuatoriana en 1527, el emperador inca, Huayna-Cápac, dirigió un ejército a la conquista de los Jíbaros (probablemente shuar o aguarunas) que vivían en la región conocida como 'Bracamoros' (...) recibió tan feroz resistencia que Huayna-Cápac tuvo que escaparse vergonzosamente, regresándose a las cordilleras andinas (Harner, 1978, p. 15).

No hay datos precisos sobre sus fronteras ya que los incas no desarrollaron un sistema de escritura ${ }^{2}$, así que la mayor parte de la información proviene de estudios arqueológicos o de cronistas del periodo colonial. Hyslop (1998) señala que las primeras aportaciones

2 A pesar de no tener escritura desarrollaron un sistema administrativo eficaz basado en el Khipu (Brokaw 2010). 
para definir las fronteras del Tawantinsuyu están estrechamente relacionadas con el sistema de caminos inca. La vía principal de este sistema se denomina Capac Ñan.

La integración de las naciones andinas en términos físico-espaciales, se establece a través de la implementación de una importante red vial (Capac Ñan), acompañada del control político administrativo de los principales centros poblados (...) la difusión de un idioma común (quechua), la movilización de contingentes de población (mitmajkuna), para tareas de cooperación y asistencia técnica, y de prácticas festivo rituales (Lozano, 1991, p. 57).

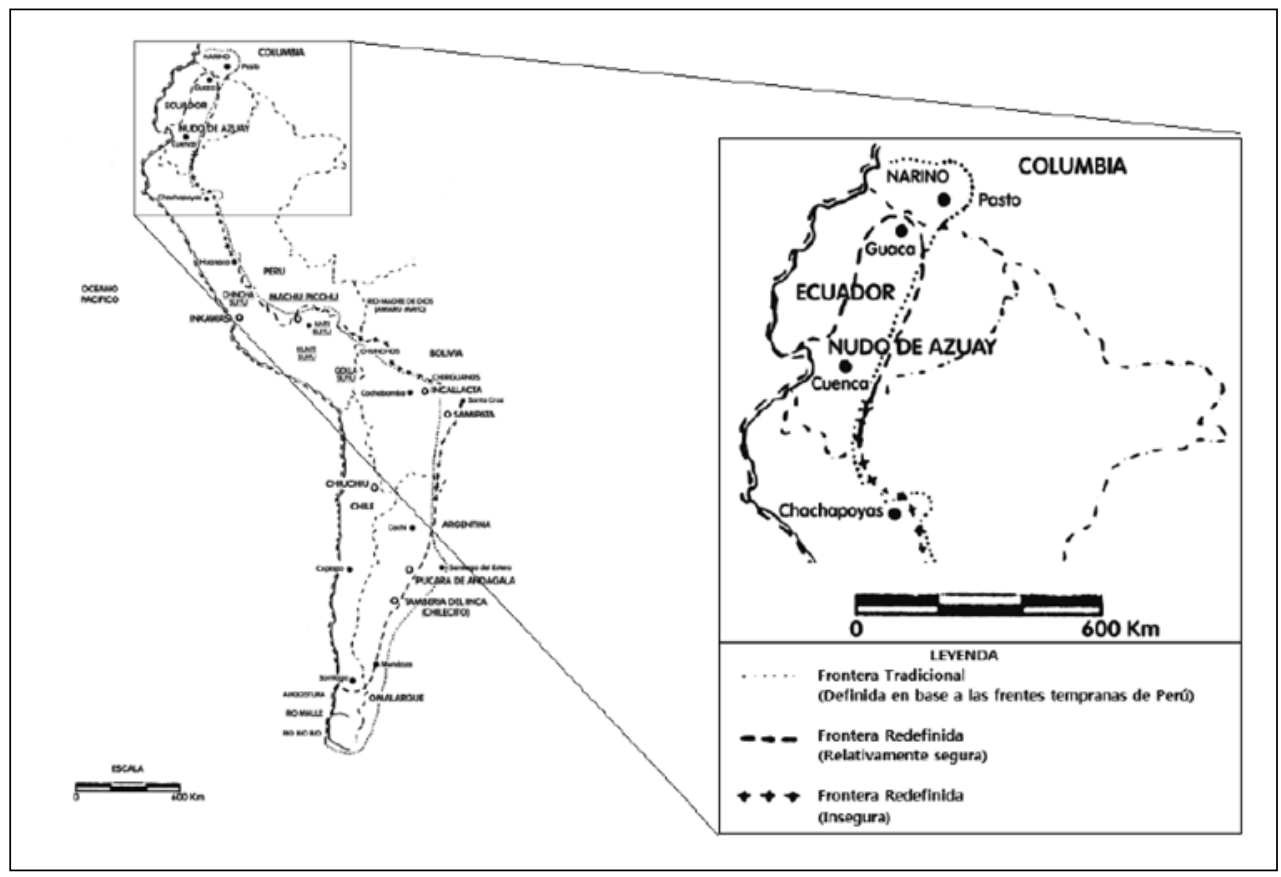

Figura 1. Fronteras del norte del Tawantinsuyu.

Fuente: elaboración propia a partir de Hyslop (1998, p. 9).

Durante la expansión incaica (1000-1500 d.C.) se crearon varios estados de frontera y en ocasiones se debían establecer formas de control tanto social, político como económico, distintas a las que se usaban dentro del Tawantinsuyu. A estos lugares fronterizos se enviaban mitmakuna para que los integrasen dentro del Estado inca. Este proceso se realizó en algunas ocasiones por medio de conquistas militares, y en otras, por la colaboración de los propios pueblos asimilados (Galindo et al., 2007). En este último caso, los incas reemplazaron a las autoridades político-religiosas o permitieron al gobernante local continuar bajo el mandato del Inca (Dillehay y Netherly, 1998, p. 8). Los pueblos asimilados eran incorporados al ejército inca para ser llevados a las fronteras y hacer la función 
de los mitmakuna (Galindo et al., 2007). Una frontera en eterna disputa correspondía a la del territorio jíbaro ${ }^{3}$ (actual frontera de Ecuador y Perú) en la cual se establecieron varias colonias de mitmakuna sin éxito alguno (Girot, 1990; Hyslop, 1998). Incluso el sistema de caminos inca que iba desde Cuzco a Quito se desviaba de este territorio (Girot, 1990). Si se observa el mapa anterior, el territorio Chicham — marcado con cruces- es una zona insegura.

Los incas también ocuparon los territorios de la Costa, pero el control no fue total (Ayala, 2008). Existe escasa información sobre los indígenas que allí radicaban, aunque se sabe que en la época de la conquista hispánica había una gran diversidad de pueblos según los escritos de la época (Romoli, 1963, p. 263). Las poblaciones de la Costa intercambiaban la sal por los productos de tierra fría y tenían relación ocasional con los mercaderes de lo que actualmente correspondería al territorio peruano (Romoli, 1963, p. 285).

En cuanto a las fronteras entre los diversos ámbitos geográficos y las relaciones existentes entre los pueblos andinos y las poblaciones de la Amazonía y de la Costa hay opiniones dispares. Según Yampara (2005, p. 40) los pueblos andinos vivían "en complementariedad armónica con las tierras bajas (Pacífico y Amazonía) (...) en agnados prehistóricos", mientras que Hyslop (1998, p. 37) señala que "los incas consideraban incivilizados a los indios de las tierras bajas tropicales", y que "el imperio construyó fortalezas en algunas partes de la frontera oriental para resguardarla de los "bárbaros" de las tierras bajas"4. En la misma línea que Yampara, Turpo (2006, p. 164) señala que el Tawantinsuyu no se construyó por medio de la conquista sino a través de un "pacto social comunitario a partir de su lógica de pensamiento cósmico".

Sin embargo, varios autores señalan que las relaciones entre las poblaciones amazónicas y andinas que existían desde tiempos inmemoriales se complicaron debido al aumento de las hostilidades durante 1000 y 1500 d.C., periodo que coincide con la expansión del Tawantinsuyu (Santos-Granero 2004, p. 179). En este sentido, aunque fue la conquista hispánica la que reafirmó la existencia de una frontera entre las poblaciones de la Costa y de la Sierra con las de la Amazonía, fueron los incas los que dieron los primeros pasos en reafirmar esa división (Benítez y Garcés; 1998; Taylor, 1994).

[E]l proceso de ruptura entre las tierras altas y las bajas, ya lo habían empezado los incas; estos desmantelaron en gran medida el sistema de alianzas políticas verticales, por ejemplo, y fueron los primeros en formular la dicotomía entre la "civilización de las tierras altas y la 'barbarie' de la selva”' (Taylor, 1994, p. 23).

3 Jíbaro es un término colonial que a día de hoy se sigue utilizando para referirse a los pueblos indígenas Achuar, Shuar, Wampis, Awajun y Shiwiar. Evitaremos usarlo sustituyéndolo por Chicham, tomando en cuenta la forma en la que se autoreconocen lingüísticamente. Aun así, respetaremos la denominación si ha sido utilizada en las citas literales.

4 Rostworoski (1988) considera que el Tawantinsuyu no fue un imperio sino un Estado. Turpo Choquehuanca $(2006,2011)$ lo denomina Estado Plurinacional Confederado de Pueblos y Naciones. 
Aunque en el presente trabajo hemos dividido las fronteras y los pueblos indígenas en tres ámbitos (Sierra, Costa y Amazonía) "es sumamente importante recalcar el hecho de que, antes de la conquista incaica, había una cierta integración entre las tres regiones geográficas, que de ninguna manera se hallaban aisladas" (Benítez y Garcés, 1998, p. 117). En este sentido, concluye Colajanni (2005, p. 261) que entre aquellos pueblos existían formas de "relaciones internacionales" y Beier (2007, p. 123) señala que los sistemas de relaciones comerciales unían a muchas personas en un sistema internacional no occidental que funcionaba en su totalidad.

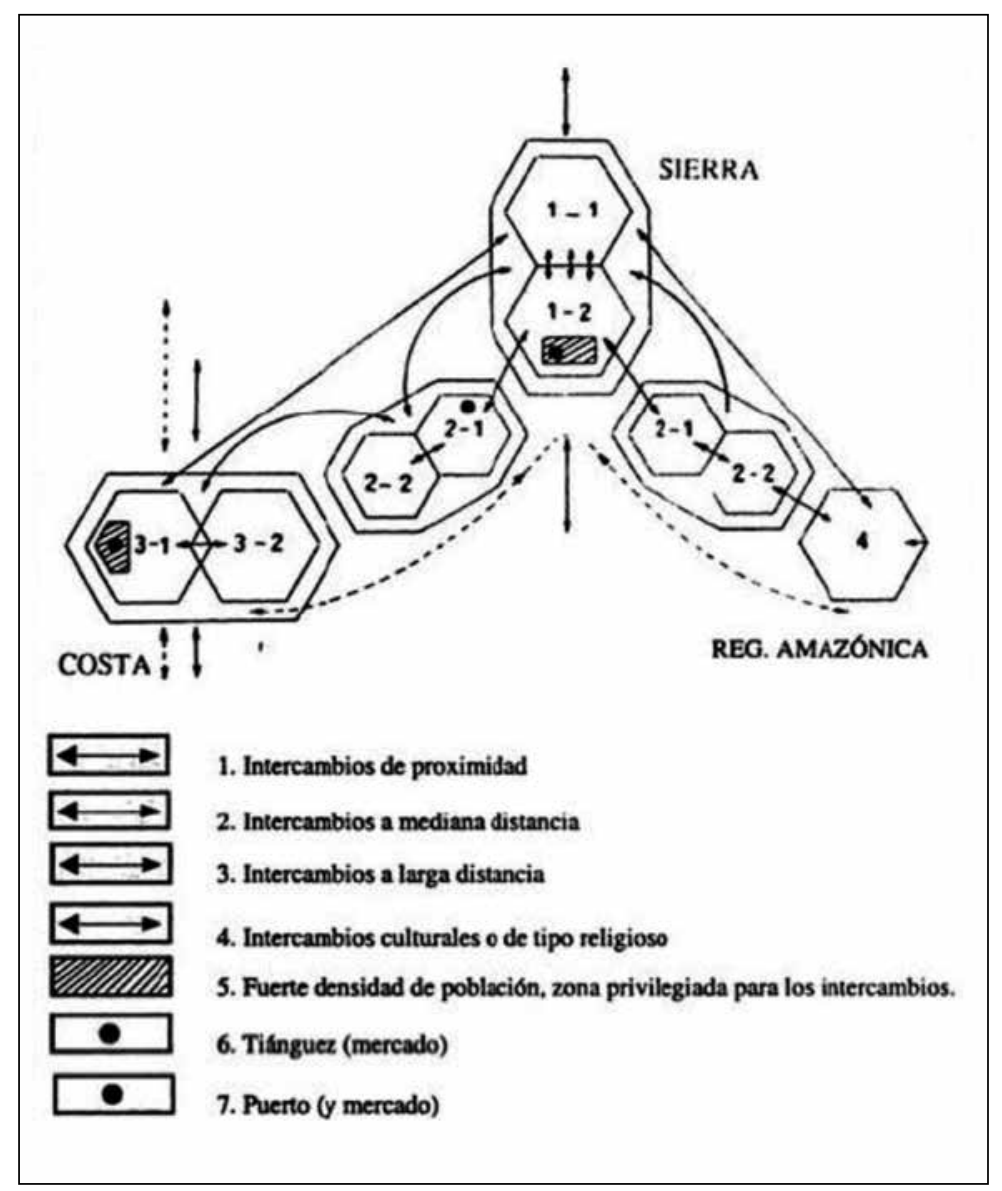

Figura 2. Esquema espacial de los intercambios.

Fuente: Benítez y Garcés (1998, p. 119).

Los primeros escritos de los cronistas del periodo colonial destacan la figura de los mindalaes que realizaban los intercambios de larga distancia (Renard-Casevitz, Saignes y Taylor 1988; Salomon, 1978). Uno de los bienes intercambiados en la larga distancia era 
el mullu (Spondylus), un molusco bivalvo de aguas calientes del Pacífico, considerado un objeto de culto entre los incas (Hocquenghem, 1993). Estas formas de comercio interétnico comenzaron a desaparecer a medida que se fueron expandiendo las fronteras de la conquista ibérica sobre el Tawantinsuyu (Caillavet, 2000).

\section{El cristianismo, los Imperios y la expansión colonial}

Durante el siglo XV había una gran disputa entre los Imperios de Europa - como la existente entre la Corona de Castilla y Aragón, y la Corona de Portugal— con el objetivo de obtener las rutas de la especiería hacia India. Portugal obtuvo ventaja ya que el Papa expidió las bulas Romanus Pontifex (1455) e Inter Caetera (1456), luego ratificadas por el Tratado Alcazobas-Toledo en 1480, que daban a Portugal el derecho a conquistar la mayoría de los territorios del mundo conocido hasta aquel momento en Europa. En este sentido, la Corona de Castilla y Aragón se vio limitada a las islas Canarias y al Atlántico por lo que autorizó la partida de Cristóbal Colón hacia las Indias rodeando el globo (Fernández, 1990).

Cuando los Reyes Católicos recibieron la noticia del 'descubrimiento' de la ruta transatlántica hacia las 'Indias', decidieron utilizar las bulas papales para ratificar su derecho sobre los nuevos territorios. De modo que en 1493 se emitieron las bulas Inter Caetera que fueron las que dividieron el mundo entre los dos imperios. A los Reyes Católicos les corresponderían entonces las tierras que quedaban desde Cabo Verde y las islas Azores hacia el oeste y a Portugal las tierras del este. Tras las bulas papales tuvieron lugar otros acuerdos internacionales entre los dos imperios de la península ibérica para la repartición de territorios que resultaron fundamentales para redefinir sus fronteras, como lo fueron el "Tratado de Tordesillas, sustituido por el Tratado de Madrid, en 1750, perfeccionado posteriormente por el Tratado de San Ildefonso, en el año 1777” (Muratori, 2007, pp. 22). $\mathrm{Al}$ respecto, Lucena igualmente aclara que:

El Tratado de Madrid supuso un hito tanto para la diplomacia hispano-portuguesa como para el marco tradicional de las relaciones entre las diferentes potencias europeas. (...) En el campo jurídico las innovaciones fueron enormes (...) se aplicó el principio del derecho civil romano —el uti possidetis - al campo de las relaciones entre estados, una esfera de derecho público. El 'uti possidetis iuris' de Tratado de Tordesillas se transformó en 'uti possidetis facti' una pirueta jurídica que permitió la legalización de todo el proceso de ocupacion portugues (Lucena 1993, pp. 81-82).

Tras la repartición del mundo entre los dos imperios la primera división del territorio que realizó la Corona de Castilla y Aragón en América fue a través de la capitulación. La capitulación consistía en un contrato entre un particular y la Corona que contaba con el visto bueno del Papa (Balutet, 2007). De esta manera, los conquistadores a los que se les había asignado la capitulación tenían el poder de repartir las tierras y de explotarlas 
(Aymerich, 2003, p. 384). A cambio, debían difundir el evangelio entre la población indígena y es por este motivo que se enviaron sacerdotes con los conquistadores (Balutet, 2007). Los primeros conquistadores en llegar a América eran campesinos sin educación de Castilla, Extremadura o Andalucía que se amotinaban con facilidad y no tenían respeto por la autoridad (Bradshaw, 2005).

\section{La conquista de la Sierra, Costa y Amazonía}

A la llegada de los primeros conquistadores a Tumbes ${ }^{5}$ — Francisco Pizarro y Diego de Almagro- encontraron el Tawantinsuyu en plena guerra civil entre Wascar y Atawalpa, hijos de Wayna Kapak, que había muerto en 1527. Al primero de ellos, lo apoyaban desde Cuzco, al segundo desde Quito, es decir, el Tawantinsuyu se encontraba dividido en dos regiones, una al norte y otra al sur. Pizarro y Almagro tomaron ventaja de la guerra para conquistarlos en poco tiempo y tuvieron vía libre para entrar dentro de las ciudades y pueblos indígenas aliados. Sobre este tema Turpo muestra una visión muy distinta:

Los intelectuales colonizados y colonizadores mestizos y criollos explican, por ejemplo, que Waskar y Atawalpa se encontraban en 'guerra civil' por el 'poder imperial', lo que facilitó la invasión espańola (...) Esta insinuación no es otra cosa que un burdo justificativo para continuar su loca carrera genocida, sobre todo, para hacer válido las acusaciones que se lanzó contra el Inka Atawallpa. Entre ellas está el hecho de que Atawallpa era hijo natural y Waskar hijo legítimo, en consecuencia, el único heredero del poder imperial. Ahora bien, es importante preguntarse si estos valores católicos de lo natural y legítimo son valores que se recreaban en la civilización tawantinsuyana" (Turpo, 2011, p. 54).

Esto último, se puede observar tanto en Guamán Poma de Ayala y Garcilaso de la Vega que consideran que Atawalpa no era el legítimo heredero, y tanto él como Rumiñahui, guerrero indígena fiel a Atawalpa que luchó contra los peninsulares, son caracterizados como mezquinos y ruines por los mencionados cronistas (Estupiñan, p. 2006).

Por órdenes de Pizarro asesinaron a Atawalpa en Cajamarca, a pesar de haber recibido la recompensa solicitada para su liberación (Poma de Ayala, 1980, pp. 275-286), y después los conquistadores se dirigieron hacia Cuzco para aliarse con los incas del Sur (Regalado, 1997). De todas maneras, esta última alianza no duró mucho tiempo ya que Manko Kapac II se rebeló en contra de la Corona.

Tras la conquista de la Sierra, los peninsulares enviaron expediciones hacia la Amazonía con el fin de encontrar los tesoros de 'El Dorado' y 'La Canela'. Son numerosos los mitos acerca de las riquezas que existían en aquel lugar tanto en el periodo incaico como en el colonial (Girot, 1990). La falta de poblamiento de la Amazonía ofrecía pocos atractivos para el establecimiento colonial y por ello estas áreas de frontera para el imperio

5 Departamento ubicado actualmente al norte de Perú. 
fueron ocupadas por las misiones (Jackson, 2009). Desde el momento inicial de la conquista la sociedad colonial estableció como centros sólo los lugares que ofrecían recursos económicos inmediatos y en los que existían recursos humanos suficientes (Ibarra, 1994), es decir, mano de obra indígena y más adelante la población negra.

Cronológicamente, la entrada hacia la Amazonía — hoy correspondiente a la parte del Ecuador - tuvo lugar entre los años 1540 y 1620, y; el interés principal en aquel territorio por parte de la Corona eran las minas de oro que habían sido descubiertas (Girot, 1990; Taylor, 1994). Tras la creación de la Audiencia de Quito en 1563, se "posibilitó el ingreso a la Amazonía desde los andes septentrionales, bajando por los ríos Napo y Putumayo" (Casanova, 2000, p. 139). Fue la expedición de Francisco de Orellana la que bautizó al ecosistema con el nombre Amazonas (Trujillo, 2001), y, además, despertó las inquietudes de los misioneros que decidieron adentrase con el fin de evangelizar a sus pobladores (San Román, 1994). Aunque ya hemos señalado que el interés principal de la Corona era la explotación de las materias primas, la creación de las ciudades servía para facilitar la entrada de los misioneros hacia la Amazonía y extender el evangelio (Tapia, 2004, p. 41).

Desde la segunda mitad del siglo XVI hasta principios del XVIII no se mostró gran interés en la región amazónica debido a diversas razones. En primer lugar, ya se disponía de recursos suficientes que provenían de las minas de plata de Potosí y de oro en Nueva Granada. En segundo lugar, se desistió de pensar en la idea de que en la Amazonía había grandes riquezas, y en tercer lugar, porque las poblaciones indígenas reaccionaban con hostilidad a la llegada de los conquistadores (Torres-Londońo, 2012). De este modo, los asentamientos selváticos fueron muy escasos y fueron desapareciendo con el tiempo (Taylor 1994; Trujillo, 2001). En este sentido, Taylor (1994, p. 22) señala que "la relativa debilidad de los métodos de control y de organización de las poblaciones indígenas locales" así como "el agotamiento de los recursos auríferos" y "la desaparición de la mano de obra india que había huido, o que había sido masacrada o aniquilada por las epidemias" fueron factores decisivos para las migraciones ocurridas desde la Amazonía hacia la Sierra.

La mayor consecuencia de estos factores fue que la región amazónica se trasformó en un lugar de frontera en todos los sentidos del término, de una región antiguamente muy poblada en ciertas zonas y sobre todo estrechamente asociada por múltiples lazos políticos, sociales, económicos y culturales a las poblaciones de la sierra (Taylor, 1994, p. 23).

Los peninsulares también tuvieron dificultades para conquistar la Costa ya que el clima y la geografía eran adversos y se sucedían las rebeliones indígenas como las de los Sindagua (Jurado Noboa, 1990). Las poblaciones de la Costa (al igual que las del Amazonas) se escondían en los bosques para escapar del sistema colonial por lo que se alteraron sus sistemas de producción y se interrumpieron sus sistemas de intercambio. 
En la rivera del mar, las poblaciones que vivían de intercambios marítimos sufrieron trastornos de sus medios de subsistencia. Regiones enteras de la cuenca del río Esmeraldas, otrora bien pobladas y donde se practicaba una agricultura desarrollada (...), se vieron reducidas prácticamente a nada, al punto de convertirse, varios siglos más tarde, en regiones de colonización (Portais, 1983, p. 88).

La conquista de la Costa al norte del actual Ecuador no se realizó hasta principios del siglo XVII, momento en el que se crearon las ciudades de Barbacoas, Iscuandé y Tumaco (Wade, 2002, p. 4; Jurado Noboa, 1990). Aunque en el siglo XVII había esclavos africanos para explotar las minas de Barbacoas (Ayala, 2008), no existía una fuerte presencia estatal ya que su integración a Estado no fue hasta bien entrado el siglo XVIII (FernándezRasines, 2001). Al parecer, los Malabas y Tomolos vivían en Esmeraldas a la llegada de los conquistadores; que tras varios levantamientos entre 1611 y 1619 desaparecieron ya que no se encontraron más escritos sobre ellos (Albornoz Peralta, 1971).

\section{Los procesos de resistencia indígena en las fronteras}

Los líderes de los pueblos indígenas no aceptaron de forma pasiva este nuevo sistema de organización social, económica y política impuesto por la Corona. Por esa razón surgieron varias rebeliones y procesos de resistencia en la Sierra, Costa y Amazonía a lo largo del periodo colonial. La vinculación entre la resistencia indígena y los ataques a los europeos se encontraba ligado a las zonas de frontera (frontiers) existentes en los límites del imperio. Estas fronteras en América del Sur coincidían en su gran mayoría con los vastos espacios de la Amazonía aún desconocidos, o los territorios de la Costa. Como ya se señaló, estos límites entre lo conocido y lo desconocido fueron ocupados principalmente por las misiones.

La frontera del Tawantinsuyu y después la de la Colonia sólo penetró parcialmente en los vastos llanos orientales, de modo que muchos grupos étnicos permanecieron allí con sus formas tradicionales de vida como si Colón y Pizarro no hubieran llegado a estas latitudes. Una de las excepciones más notables fueron las misiones-reducciones jesuíticas que, en nuestra región, cubrían los territorios amazónicos de Maynas (entonces dependientes de Quito, hoy del Perú) ... (Albó 2002, p. 176)

El territorio que iba más allá de estos límites cumplió dos funciones principales. En primer lugar, funcionó como vía de escape para los pueblos indígenas insurgentes que escapaban de las encomiendas y de las misiones. Hubo lugares que fueron zonas de refugio desde el periodo colonial hasta la mitad del siglo XX (Girot, 1990, p. 130), de modo que "en las zonas fronterizas, las poblaciones indígenas nunca se sometieron por completo; más bien aprovecharon los inmensos territorios de los que disponían para retroceder ante el avance de los colonos" (Manrique, 2006, p. 57). 
En segundo lugar, estos espacios de frontera los aprovechaban los indígenas considerados salvajes o aucas para atacar las ciudades construidas por los peninsulares (Bernand, 2003, p. 181). La ciudad de Jaén, por ejemplo, tuvo que ser reubicada en varias ocasiones por el temor hacia el ataque de los pueblos Chicham (Girot, 1990, p. 127). Según Manrique (2006, p. 58) durante el periodo colonial ocurrió "una intermitente e interminable guerra de fronteras (...) en la que las grandes poblaciones españolas nunca estuvieron amenazadas seriamente". Esta afirmación es hasta cierto punto cuestionable debido a que existieron grupos indígenas que presentaron una gran resistencia - creando una frontera - frente al sistema colonial, como fue el caso del mencionado alzamiento de los Chicham del siglo XVI o el de los incas y aymaras de la Sierra del XVIII.

En la región andina un espacio de frontera aceptado hasta cierto punto por los españoles correspondía a Vilcabamba, el último reducto inca. Los conquistadores, en un primer momento, proclamaron emperador inca a Tupak Walpa y después a Manko Kapak II tras la muerte del primero (Pérez Marcos, 2002). Se podría decir que su nombramiento correspondía a un acto simbólico más que real, ya que no era más que una marioneta de los conquistadores. Pero alrededor de 1536 Manko Kapak II se rebeló ante las injusticas que sufrían los indígenas, y él y su ejército lograron sitiar la ciudad de Cuzco (Regalado, 1997; Renard-Casevitz, Saignes y Taylor, 1988, p. 133). Tras varios encuentros bélicos detallados por Regalado (1997, pp. 28-38) los incas fueron derrotados por los conquistadores, lo que provocó que Manko Kapak II se retirase a Vilcabamba, donde él y sus sucesores resistieron hasta 1572 (Renard-Casevitz, Saignes y Taylor, 1988; Bernand, 2003).

Hacia 1545, en medio de extrañas circunstancias, Manco Inca terminó muerto por un grupo de antiguos soldados almagristas que estaban hospedados en su propia morada. Pero el espíritu de la resistencia quechua fue mantenido por sus hijos, quienes, conscientes de la nueva realidad política de su patria, se animaron a efectuar negociaciones con las autoridades virreinales (Hampe, 2000, p. 84).

Entre las negociaciones más destacables entre los incas y la Corona, encontramos la 'Capitulación de Acobamba' firmada en 1566, tratado por el cual en Vilcabamba "se establecía la paz y se perdonaban los delitos cometidos por los indios rebeldes, bajo la condición de permitir el asentamiento de un corregidor y de frailes doctrineros" (Hampe, 2000, p. 85). Esta capitulación fue acordada entre Titu Kusi Yupanki, tercer monarca de Vilcabamba, y el Gobernador Lope García de Castro el 24 de agosto de 1566, mediante la Cédula del 14 de octubre, y se ratificó y complementó en julio de 1567 (Levaggi, 2002, pp. 55-61).

El sucesor del Gobernador García de Castro fue el Virrey Francisco de Toledo que interpretaba la Capitulación como una claudicación de la Corona (Levaggi 2002, p. 62). En Vilcabamba el sucesor de Titu Kusi fue Tupak Amaru, último inca de Vilcabamba que ascendió al trono en 1571 (Hampe, 2000). Pérez Marcos (2002, p. 1335) señala lo siguiente sobre la capitulación de Acobamba y la conquista de Vilcabamba: 
Thupa Amaru (...) partía del desconocimiento de los términos de la capitulación de Acobamba, cerrando las fronteras como respuesta desafiante al virrey Toledo quien ignorando la muerte de Titu Cussi envió a Vilcabamba al dominico fray Gabriel de Oviedo, en julio de 1571 para que entregara al inca los documentos confirmatorios de la negociación de Acobamba (...), pero encontró cerrada la frontera y hubo de volverse, lo que desencadenó la cólera del virrey, que escribió a Tito Cussi (creyéndole vivo) una amenazadora carta (...) Esta carta no llegó a su destino por desencadenarse la guerra que acabaría con la ocupación de Vilcabamba... (Pérez Marcos, 2002, p. 1335).

Finalmente, Tupak Amaru (luego conocido como Tupak Amaru I para diferenciarlo de Tupak Amaru II) fue decapitado públicamente en Cuzco por órdenes del Virrey Toledo (Kubler, 1947, p. 202) y así concluyó la dinastía de los incas de Vilcabamba. A pesar de ello, durante los siglos XVIII y XIX las rebeliones indígenas comenzaron a multiplicarse en el Virreinato de Perú. En la Audiencia de Quito Moreno (1976) ha recopilado al menos diez casos de levantamientos indígenas que tuvieron lugar desde 1730 hasta 1803 . Albornoz Peralta (1971) también recoge los levantamientos indígenas del Ecuador estableciendo una división entre aquellos de la Costa, de la Sierra y de la Amazonía en este periodo.

El último gran levantamiento indígena durante el periodo colonial en los Andes y quizás el más conocido a día de hoy, tuvo lugar entre los años 1780 y 1782: el levantamiento de Tupak Amaru II y Micaela Bastidas (Quechua) y el de Tupak Katari y Bartolina Sisa (Aymara). ${ }^{6}$ Aunque esta rebelión no se dio en las fronteras de la Colonia podría calificarse como rebelión transfronteriza (Escárzaga, 2004) ya que traspasó los límites virreinales. Este levantamiento fue duramente reprimido ya que tras él "se prohibió la enseńanza en quechua, la escenificación de dramas en esa lengua y la reedición del texto fundador del nacionalismo inca, los Comentarios de Garcilaso" (Manrique, 2006, p. 63). Aunque esta rebelión transfronteriza no tuvo éxito, resultó fundamental para la lucha anticolonial (Buron-Brun, 1999) y condicionó el proceso de independencia (Manrique, 2006, pp. 5960) que sería retomado pocos años después por los criollos.

En cuanto a la Amazonía, a finales del siglo XVI el primer levantamiento de gran magnitud tuvo lugar en la región de Quijos. Al parecer los Quijos eran un grupo formado por diversos indígenas de la Amazonía y de la Sierra, conocidos también con el nombre Sumacos o Kichwas del Oriente (Benítez y Garcés, 1998). El alzamiento de los Quijos fue principalmente una respuesta contra los abusos cometidos a través del sistema de la encomienda. Las primeras insurrecciones tuvieron lugar a partir de 1560 (Ospina, 1992)

6 "Asociado al nombre de Túpac Amaru encontramos siempre el nombre de su mujer: Micaela Bastidas. Éste tampoco es un hecho casual. Prácticamente en todas las rebeliones del periodo es posible ubicar, al lado de los grandes caudillos, sus complementos femeninos. Así en la rebelión del Alto Perú paralela a la de Túpac Amaru, dirigida por Túpac Catari, destacan las figuras de dos mujeres: Bartolina Sisa, esposa y Gregoria Apasa, hermana del jefe (...) Micaela Bastidas, así como las demás mujeres rebeldes, no fueron simples figuras decorativas al lado de un gran caudillo. Por el contrario: representaban la expresión más radical de las rebeliones" (Mires, 2005, p. 32) 
pero no fue hasta la década siguiente que los peninsulares entraron en guerra directa con los Quijos para pacificar la zona. La segunda rebelión, en el año 1579, se conoce también como la de los 'pendes' o los 'shamanes indígenas' liderados por Jumandi (Oberem, 1980; Muratorio, 1981; Ruiz Mantilla, 1992). Jumandi, en alianza con otros líderes indígenas, destruyó las ciudades de Ávila, Archidona y Baeza (Albornoz Peralta, 1971). Finalmente, los conquistadores los reprimieron militarmente, y más adelante, los jesuitas utilizaron "esta vía como paso hacia la provincia de Mainas" (Trujillo, 2001, p. 21).

Cerca de Quijos ocurrió otro levantamiento en 1599, el de los Chicham, liderados por el indígena Quiruba. Tomaron las ciudades de Logroño de los Caballeros, Sevilla del Oro, Valladolid, Huamboya y Zamora, así como otros asentamientos mineros y los destruyeron (Albornoz Peralta, 1971). Pocos años antes del levantamiento se descubrieron unas minas de oro en Ayambis y Zaruma, en el territorio de los Chicham, motivo por el cual se incrementaron los malos tratos hacia los indígenas para recaudar el mayor oro posible. Los indígenas inconformes con la actitud de los peninsulares decidieron revelarse y atacaron esas ciudades como respuesta a esos abusos (Abad González 2003, pp. 112113). Aunque tras estos ataques los territorios amazónicos comienzan a abandonarse y a convertirse en una frontera, los peninsulares llevaron a cabo las cacerías de jibaros a partir de 1619, hasta que en 1705 finalmente fueron prohibidas por el Virrey de Lima (Abad González, 2003). La influencia del levantamiento fue tal que se extendió hacia las otras zonas de la Amazonía y del piedemonte amazónico.

La ruina de este gobierno causó, como consiguiente mui natural, la de Yahuarzongo, provocada i consumada por los mismos jíbaros, i luego la de Quijos i Jaen. La catástrofe se extendió hasta las tribus de Popayan que, alentadas por el ejemplo de las del sur, cometieron por allá destrozos lamentables (Fermín Cevallos, 1870, p. 148).

Posiblemente, una de las catástrofes que Cevallos (1870) menciona sobre Popayán corresponda con el levantamiento de los indígenas Sindagua en la zona fronteriza de la Costa de la actual Colombia y Ecuador. Los alzamientos de los Sindagua ocurrieron entre 1628 y 1635 (Jurado Noboa, 1990, pp. 72-75), periodo que coincide con los ataques de los peninsulares para conquistar aquellos territorios (Herrera, 2009, p. 68). Aunque desde 1579 existían encomiendas en la actual frontera de los Estados mencionados, los Sindagua representaron "una frontera donde los naturales permanecían en pie de guerra contra unos pocos encomenderos que no tuvieron el apoyo necesario para conquistar, efectivamente, la región" (Zuluaga y Romero, 2007, p. 114).

Quince años después de la victoria de los Sindagua sobre los peninsulares ocurrida en 1615 (Díaz del Castillo, 1987, p. 293 En: Jurado Noboa, 1990, p. 69) fueron arrinconados en Barbacoas. Tras esta guerra se aplicaron castigos severos con el fin de que la zona pudiera ser pacificada (Díaz del Castillo, 1938 En: Montoya Guzman, 2011). Según Montoya Guzmán (2011) la conquista del pueblo Sindagua no fue realizada solamente mediante la guerra sino a partir del establecimiento de las misiones en aquel territo- 
rio. Mientras que del lado de lo que corresponde actualmente a Colombia fue posible la conquista y posterior creación de ciudades en la provincia de Barbacoas, en Esmeraldas (Ecuador) no ocurrió hasta el siglo XX (Montoya Guzmán, 2011, pp. 18-19).

\section{La alteración de las estructuras indígenas y las fronteras sociales}

A través de la conquista se estableció en la Colonia una división del trabajo con base en la raza de las poblaciones. De este modo los estratos superiores los ocupaban los blancos, mientras que los inferiores eran ocupados por los pueblos indígenas. Hasta 1492 no existían las categorías de indios, blancos, negros, etc.; éstas fueron impuestas por la dominación colonial y como forma de expresión del poder (Germaná, 2005), haciendo coincidir "las fronteras de origen étnico y las categorías jurídicas" (Manrique, 2006, p. 46).

Además de alterar las estructuras precolombinas, se creó una categoría panindígena - la de los indios - que les otorgaba un nombre genérico que difuminaba sus diferencias y facilitaba su evangelización (Trujillo 2001, p. 78). Para la evangelización de estos pueblos los misioneros escogieron las lenguas de mayor difusión. De este modo, se estableció el Kichwa como instrumento unificador y como lengua franca además del castellano (Albó, 2002; Grisales, 2000).

La economía incaica y su organización del trabajo basada en el colectivismo sufrieron una gran alteración a medida que se fueron agotando los recursos auríferos saqueados por los peninsulares, en el sentido que llegó a alcanzar el sistema productivo por completo. Este choque de concepciones de organización del trabajo supuso un desastre para las poblaciones de los Andes (Ribeiro, 1992, p. 129). Los que habitaban la Costa y la Amazonía pudieron gozar de mayor libertad, aunque sujetos siempre al avance de la frontera colonial.

\section{El nuevo ordenamiento territorial: marcar, dividir y repartir}

En un primer momento, la división de América Latina fue realizada con base en la institución de la capitulación, de modo que la Corona con el apoyo de la Iglesia establecía los límites que se conquistarían. Pero la Corona comenzó a temer por el creciente poder que abarcaban los conquistadores debido a sus ansias de riqueza - lo que podría causar intentos de independencia- así que estableció el sistema político de las Audiencias y Virreinatos (Manrique 2006).

Así como las estructuras económicas y sociales se transforman en el curso de la historia, las estructuras espaciales evolucionan igualmente y existe una interacción dialéctica compleja entre las modificaciones de lo económico, lo social, y lo espacial. En cada época de la historia la sociedad imprime, así, su sello más o menos profundo en el espacio, pero en un espacio ya parcialmente estructurado en cuanto heredado (Deler, 2007, p. 135). 
El Tawantinsuyu fue abolido por el establecimiento del Virreinato de Perú en 1542, aunque después se creó un Estado Neoinka en Vilcambamba. La Audiencia de Quito se fundó en 1563 dentro del Virreinato de Perú7. La Audiencia de Quito fue creada con sus cuatro gobernaciones: Yaguarzongo y Pacamoros; Quijos, Sumaco y la Canela; Atacames (o Esmeraldas); y Popayán. Entre los siglos XVII y XVIII surgieron otras dos, Maynas y Jaen de Bracamoros en la región de la Amazonía, y a partir de 1638 se empieza a extender el nombre Maynas hacia todos los territorios del Alto Amazonas (Abad González 2003, p. 114).

La Corona tomó en cuenta la distribución de las diferentes poblaciones indígenas para ordenar el territorio, es decir, "la configuración de las fronteras administrativas coloniales tuvo en cuenta las diferencias establecidas por esos ordenamientos sociopolíticos para trazar las fronteras provinciales y regionales" (Manrique, 2006, p. 23). Por ejemplo, en el Oriente de la Audiencia de Quito, se crearon las gobernaciones de Quijos, Yaguarzongo, Pacamoros y Maynas, correspondientes a los nombres de los pueblos indígenas que allí vivían (Castro Ponce, 2005). Pero hay que tener en cuenta que estas denominaciones eran el nombre que los peninsulares habían decidido darles.

\section{Las instituciones socio-económicas de la conquista}

La imposición del sistema de administración colonial supuso la creación de nuevas escalas de control social (Howitt, 1993) mediante las cuales se conseguía hacer una distinción entre la 'República de Blancos’ y la 'República de Indios'. De modo que "para demostrar su conquista de espacios, se funda una nueva población creando límites entre indios y españoles, es decir, se elabora una jerarquización del espacio conquistado" (Trujillo, 2001, p. 20). La Sierra fue el espacio donde la colonia se estableció con más fuerza en comparación a la Costa y la Amazonía, debido a la importancia demográfica y su rápida asimilación (Cebrián, 1999, p. 42).

A pesar de que estas nuevas escalas operaban como formas de control social, favorecieron indirectamente a que se siguieran manteniendo las autoridades políticas indígenas en las comunidades (Manrique, 2006, p. 57). La nobleza indígena, representada por los kurakas, estableció pactos con los encomenderos para recoger los tributos. En este sentido, los kurakas establecían el vínculo entre la sociedad colonial y la 'República de Indios'.

Las instituciones socioeconómicas principales para establecer el poder colonial y explotar la mano de obra indígena fueron la mita, la encomienda y la misión. La mita tenía su origen en el periodo incaico, pero los conquistadores alteraron su significación convirtiéndolo en un sistema de explotación. A través de la mita los pueblos indígenas debían enviar algunos de sus habitantes a trabajar para la corona con el fin de intercambiar su trabajo por el salario. En un primer momento, la mita se estableció en Ecuador en los

7 Dentro del Virreinato se crearon también la Audiencia de Lima en 1543 y la de Santa Fe de Bogotá en 1549. 
lugares donde existían minas y tras la decadencia de éstas se extendió hacia el sector agrícola (Benites, 1995, pp. 100-102).

Mediante la encomienda los conquistadores se hacían cargo de un grupo de indígenas y de sus tierras. Hubo diversas leyes promulgadas por la Corona con el fin de acabar con este sistema. Las primeras protestas surgieron a partir de 1512 por parte de unos frailes dominicos tras las que se promulgaron las Leyes de Burgos con las cuales la Corona protegía a los indígenas de la explotación. Aun así, éstas no tuvieron efecto (Benítez y Garcés, 1998). Asimismo, también se promulgaron las Leyes Nuevas en 1542 debido a la influencia de las demandas de Fray Bartolomé de las Casas (Bernand, 2003).

La amplitud del territorio amazónico hacía difícil su control y por ese motivo los gobiernos locales necesitaban del apoyo de las misiones para defender esas tierras que aún no estaban delimitadas y en ocasiones eran invadidas por los poderes vecinos. Asimismo, las poblaciones indígenas se mostraban en ocasiones hostiles y la misión era de gran ayuda para 'pacificar' a los indígenas 'salvajes' (Taylor, 1994, p. 26). Las misiones eran la institución típica de los territorios de frontera y su función coincidía con los objetivos militares de la Corona (Girot, 1990, p. 125) a pesar de que tuvieran una visión diferente de la frontera.

Los misioneros no concebían la frontera como un enclave (a diferencia de los soldados y los oficiales de la Corona), sino como una línea de comunicación entre los diferentes pueblos de indios que se pensaba fundar; éstos debían estar siempre conectados entre sí, con vigilancia de los indios amigos o los ya dominados. Los pueblos no funcionarían ya como unos puntos concretos de penetración, aislados, cerrados e inexpugnables, sino como unos puntos de avanzada. Se convertirían además en un espacio abierto a los indios. No era el lugar desde donde se emprenderían expediciones hacia la 'tierra adentro', sino un territorio al que se aspiraba dominar, con el fin de comunicarse con sus habitantes (Montoya, 2011, p. 20).

En lo que corresponde a las fronteras actuales de Perú y Ecuador se estableció la misión de Maynas. Allí se fueron creando cada vez más pueblos por influencia de los jesuitas (Codina 2005, Torres-Londoño 2012) con escasa población colona por las “dificultades para someter a los indígenas a los tributos de la encomienda y las obligaciones de la mita" (Torres-Londoño 2012). En este sentido, podríamos decir que la Amazonía constituyó una frontera entre la potencia hispánica y el resto de potencias, y la condenó "a jugar el papel de eterna frontera entre sus posesiones y las de los demás imperios" (Ibarra, 1994, p. 55).

Cuando ocurrió la expulsión de los jesuitas entre 1767 y 1768, las misiones de Maynas pasaron a manos de otras órdenes religiosas y entraron rápidamente en decadencia hasta desaparecer (Torres-Londoño, 2012) por lo que los indígenas volvieron de nuevo a la selva (Trujillo, 2001, 79). Hubo varios intentos por controlar el territorio, uno por parte de los franciscanos que sustituyeron a los jesuitas, pero no lo lograron, y otro, por el gobernador de la provincia, Requena, que tampoco lo consiguió (Santos y Barclay 2002). 


\section{Las reformas borbónicas y la redefinición de las fronteras del Imperio}

En el año 1700 los Borbones tomaron el poder de la Corona y modificaron la forma de gobierno que había llevado la dinastía de los Habsburgo. Las reformas borbónicas supusieron un cambio que reflejaba la nueva filosofía del Estado que iba a la par de los principios de la Ilustración (Romero, 2003, p. 23). Estas reformas se realizaron tanto en la metrópoli como en las colonias y se aplicaron a los campos económicos y fiscales —el tributo, la alcabala, los estancos, la liberalización y apertura comercial—, y los administrativos — militares, civiles y de ordenamiento territorial (Borchart, 1998).

Se estableció un modo de gobierno más centralista que limitó el poder de los habitantes de las colonias y consecuentemente aumentaron las rivalidades entre los americanos y los europeos (Manrique, 2006). Asimismo, el polo económico se trasladó de la Sierra hacia la Costa, ya que el decreto de liberalización del comercio a partir de 1774 benefició a Guayaquil y principalmente al sector del cacao que fue el motor de su economía (Laviana, 1987, pp. 163-226).

Aunque las reformas borbónicas estaban influenciadas por los principios de la Ilustración francesa, se realizaron también para tratar de solucionar la crisis económica y política causada por la Guerra de Sucesión (1700-1715) y especialmente por el desarrollo del capitalismo inglés, que afectaba al "comercio marítimo de las colonias españolas en América” (Romero, 2003, p. 23). De modo que por razones tanto internas como internacionales las reformas borbónicas refundaron el imperio colonial creando nuevas demarcaciones territoriales (Manrique, 2006, p. 69).

Sus causas principales fueron de orden estratégico, ya que se trataba de hacer frente a la presencia de los ingleses en el Pacífico y en Atlántico sur, así como a las incursiones de ingleses, franceses y rusos en Norteamérica. Sin embargo, estas reformas también buscaron la racionalización de los territorios coloniales en América desde el punto de vista de su administración (Romero, 2003, p. 23).

Del mismo modo, se trató de dar solución a los espacios despoblados que habían ocupado una situación periférica y fronteriza estableciéndose las unidades virreinales para mejorar su administración. Mientras que durante el reinado de los Habsburgo sólo existió el Virreinato de Perú, los Borbones crearon dos más: el de Nueva Granada y el de La Plata.

Hacia 1740 (...) una de las trasformaciones más importantes fue la aparición de una conciencia geográfica territorialista, la aceptación de que la ocupación física del continente americano y su control estatal directo debían ser parte sustancial de cualquier programa de reformas. A partir de 1750 es el propio Estado Borbónico, situado en un nuevo marco de relaciones internacionales y apoyado en un fuerte dispositivo militar y científico el que se implanta en las áreas marginales, las ordena de acuerdo con una nueva lógica de organización territorial e intenta articular un efectivo control social y político del espacio (Lucena, 1996, p. 268). 
En cuanto a las reformas del territorio que afectaron a nuestro lugar de estudio, a partir de 1717 la Audiencia de Quitó, que tradicionalmente formaba parte del Virreinato de Perú, se incorporó al Virreinato de Nueva Granada por motivos administrativos; aunque en términos eclesiásticos seguía haciendo parte del anterior. En 1722 se abolió el Virreinato de Nueva Granada por lo que la Audiencia de Quito regresó a la situación anterior a 1717, pero en 1739 se volvió a restablecer el de Nueva Granada y la Audiencia se desgajaba de nuevo del Virreinato de Perú (Maier, 1969). Esta reformulación fronteriza contribuyó a aumentar las tensiones porque algunas zonas se beneficiaron en perjuicio de otras. Por este motivo comenzaron a fortalecerse los nacionalismos regionales que después se evidenciarían en el proceso de independencia latinoamericano (Ibarra, 1994, pp. 57-58).

Por último, cabe recordar que la Cédula de 1802 emitida por Carlos IV, separó las provincias de Maynas y Quijos del Virreinato de Nueva Granada — tradicionalmente asociadas a la Audiencia de Quito- al Virreinato del Perú en términos eclesiásticos y militares (St. John 1994). Estas últimas fragmentaciones — y anexiones — son útiles para comprender las futuras reivindicaciones que harían Perú y Ecuador sobre su soberanía como Estados nacionales.

\section{Conclusiones}

En este artículo hemos podido evidenciar cómo ha evolucionado la configuración del territorio en el Virreinato de Perú, particularmente en lo que se refiere a los procesos de delimitación fronteriza. Si bien se ha realizado una reflexión panorámica sobre la expansión de las fronteras coloniales en la Sierra, Costa y Amazonia; esto permite comparar y entender de manera holística lo ocurrido en cado uno de estos tres espacios.

En el trabajo se constata que los indígenas no fueron sujetos pasivos que aceptaron la conquista y la explotación, sino que articularon diversas formas resistencia ante los europeos. Estas formas de resistencia las podemos ubicar precisamente en las fronteras, donde el dominio colonial trató de expandirse, apropiar nuevos territorios y colonizar nuevos sujetos. En este sentido, hubo diversos levantamientos indígenas en la Costa, Sierra y Amazonia.

Las fronteras (frontiers) como zonas de resistencia y relaciones de poder eran utilizadas por los grupos indígenas para atacar los asentamientos coloniales o para escapar de la explotación. Esto ocurría principalmente en los territorios selváticos, mas no en los serranos. Por esta razón, estos lugares fueron ocupados por misioneros, en vez de por encomenderos, con el fin de administrar el territorio. En términos generales, la Sierra fue el primer espacio en ocuparse ya que había más población y, por lo tanto, más mano de obra. La Amazonia fue convertida en una frontera y su mayor parte no fue ocupada a excepción de Maynas. 
El aparato jurídico de Europa, basado en bulas, capitulaciones y posteriormente, virreinatos y audiencias, justificaba la conquista estableciendo nuevas escalas de control social y racial sobre las poblaciones autóctonas de América Latina. De esta manera el control del territorio estaba relacionado con la expansión de la frontera colonial, y de acuerdo con la fundamentación del artículo, estas fronteras fueron lugares donde se configuraban relaciones de poder. Con respecto a esta última cuestión, cabe destacar que el papel de la iglesia fue fundamental tanto en la repartición de los territorios globales, como en los procesos de civilización realizados en las áreas de frontera (frontiers).

La conquista del Tawantinsuyu y de las otras regiones alteró las estructuras de organización territorial y cosmológica de los indígenas, desmoronando también el sistema internacional de comercio no occidental desarrollado entre los pueblos de la Sierra, Costa y Amazonía. De todas formas, aunque se sustituyeron las formas indígenas de organización política, territorial y administrativa por un modelo de organización europeo, se articularon con éste para mantener las autoridades locales.

Finalmente, las reformas borbónicas supusieron el aumento de los recelos entre diversas identidades regionales o locales, es decir, el surgimiento de nacionalismos que influirían en la construcción de los futuros estados nacionales. El nuevo ordenamiento territorial impulsado por estas reformas tendría consecuencias sobre las demarcaciones fronterizas en el periodo de independencias latinoamericano y ocasionaría las disputas interestatales a partir del siglo XIX.

\section{Agradecimientos}

La autora expresa su gratitud a la Pontificia Universidad Javeriana y a la Universidad del País Vasco por su apoyo en la elaboración de este artículo.

\section{Declaración de divullgación}

La autora declara que no existe ningún potencial conflicto de interés relacionado con el artículo.

\section{Financiamiento}

La autora no declara fuente de financiamiento para la realización de este artículo.

\section{Sobre la autora}

Minerva Campion Canelas, es licenciada en Periodismo y magister y doctorado por la Universidad del País Vasco Actualmente, es docente del Departamento de Ciencia Política de la Pontificia Universidad Javeriana. 


\section{Referencias}

Abad González, Luisa (2003). Etnocidio y resistencia en la Amazonía peruana. Cuenca: Ediciones de la Universidad de Castilla-La Mancha.

Albó, Xavier (2002). Pueblos indios en la politica. La Paz: CIPCA.

Albornoz Peralta, Oswaldo (1971). Las luchas indigenas en Ecuador. Guayaquil: Editora Claridad.

Ayala, Enrique (2008). Resumen de la Historia de Ecuador. Quito: Corporación Editorial Nacional.

Aymerich, Ignacio (2003). Derecho Latinoamericano. En Lecciones de derecho comparado, coord. Manuel Guillero Altava, pp. 381-391. Castelló de la Plana: Publicacions de la Universitat Jaume.

Balutet, Nicolás (2007). Ensayos sobre la historia española (1516-1939). Lyon: Editions Confluences y Traverses.

Beier, J. Marshall (2007). International affairs: Indigeneity, Globality and the Canadian state, Canadian Foreign Policy Journal, 13(3), 121-131.

Benites, Leopoldo (1995). Ecuador: drama y paradoja. Quito: Colección Ensayo-Libresa

Benítez, Lilyan y Alicia Garcés, A. (1998). Culturas ecuatorianas ayer y hoy. Quito: Ediciones Abya Yala.

Bernand, Carmen (2003). Impérialismes ibériques. En Le livre noir du colonialisme. XVI et XXI siècle: de l'extermination à la repentance, ed. Marc Ferro, pp. 180-236). Paris: Éditions Robert Laffont.

Borchart, Christiana (1998). La Audiencia de Quito. Aspectos económicos y sociales (Siglos XVI-XVIII). Quito: Ediciones del Banco Central del Ecuador/ Ediciones Abya Yala.

Bradshaw, Roy (2005). Redefining the nature and functions of boundaries: a South American perspective. En Holding the Line: Borders in a Global World, eds. Heather Nicol e Ian Townsend-Gault, pp. 180196. Vancouver: UBC Press.

Brokaw, Galen (2010). A History of the Khipu. Cambridge: Cambridge University Press.

Buron-Brun, Benedicte (1999). Mujeres peruanas el otro lado de la historia de Sara Beatriz Guardia. En Mujer, creación y problemas de identidad en América Latina, comp. Roland Forgues, 446-450. Mérida, Venezuela: Consejo de Publicaciones de la Universidad de Los Andes.

Caillavet, Chantal (2000). Etnias del Norte. Etnohistoria e historia de Ecuador. Quito: Ediciones Abya Yala.

Casanova, Jorge (2000). La misión jesuita entre los Aido Pai (Secoya) del río Napo y del río Putumayo en los siglos XVI al XVIII, y su relación con los asentamientos indígenas. En Un reino en la frontera. Las misiones jesuitas del periodo colonial, coords. Sandra Negro y Manuel Zarzal, 139-148. Quito: Ediciones Abya Yala.

Castro Ponce, María Soledad (2002). Yaguarzongos y Pacamoros. Quito: Ediciones Abya Yala.

Cebrián, Francisco (1999). La organización del espacio en el Ecuador. Cuenca: Ediciones de la Universidad de Castilla-La Mancha.

Cevallos, Pedro Fermín (1870). Resumen de la historia del Ecuador, desde su orijen hasta 1845. Lima: Imprenta del Estado.

Codina, María Eugenia (2005). Hacienda y misiones: el caso de Maynas. En Esclavitud, economía y evangelización: las haciendas jesuitas en la América virreinal, comps. Sandra Negro y Manuel Zarzal, pp. 243-262. Lima: Fondo Editorial de la Pontificia Universidad Católica de Perú.

Colajanni, Antonino (2005). Viejas y nuevas identidades indígenas en el cuadro de los procesos de globalización en la América Latina de hoy. En Identidad lingüistica de los pueblos indígenas de la región andina, comp. Ariruma Kowii, pp. 233-272. Quito: UASB/Ediciones Abya Yala. Roma: Instituto Italo-Latino Americano. 
Costa, Ravi (2007). Cosmology, mobility and exchange: Indigenous diplomacies before the nation-state, Canadian Foreign Policy Journal, 13, no 3: 13-28.

Deler, Jean Paul (2007). Ecuador del espacio al Estado Nacional. Quito: Universidad Andina Simón Bolívar, IFEA.

Díaz del Castillo, Ildefonso (1938). Sublevación y castigo de los Indios Sindaguas de la Provincia de Barbacoas, Boletín de Estudios Históricos 12, no. 86: 36-42.

Dillehay, Tom y Patricia Netherly (1998). Introducción. En Las fronteras del Estado Inca, comps. Tom Dillehay y Patricia Netherly, 3-32. Quito: Fundación Alexander von Humbolt/Ediciones Abya-Yala.

Escárzaga, Fabiola (2004). Fronteras volátiles: los aymarás de Perú y Bolivia. Guaca 1, nº1: 27-43.

Estupiñan, Tamara (2006). La manipulación del tirano llamado Rumiñahui: una imagen historiografía negativa en el largo plazo. En Mitos políticos en las sociedades andinas Orígenes, invenciones y ficciones, eds. Germán Carrera et al., 163-190. Venezuela: Editorial Equinoccio.

Fernández, Rafael Diego. 1990. Proceso jurídico del descubrimiento de América (Bulas, Tratados y capitulaciones, Anuario Mexicano de Historia del Derecho 2: 81-114.

Fernández-Rasines, Paloma (2001). Afrodescendencia en el Ecuador. Raza y género desde los tiempos de la Colonia. Quito: Ediciones Abya Yala.

Galindo, Mario, Ramiro Bueno, Bonifacio Cruz y Elizabeth Cruz (2007). Visiones aymaras sobre las autonomias. Aportes para la construcción del Estado nacional. La Paz: PIEB.

Germaná, César (2005). La migración internacional en el actual periodo de globalización del sistema mundo-moderno/colonial, Alternativas: cuadernos de trabajo social, 13:19-3.

Girot, Pascal (1990). Resistencia indígena y la evolución de fronteras en Rio Chinchipe, alto Marañón, Revista de Historia, 21-22: 119-148.

Grisales, German (2000). Nada queda, todo es desafío: globalización, soberania, fronteras, derechos indígenas e integración en la Amazonía. Bogotá: Convenio Andrés Bello.

Hampe, Teodoro (2000). Guerras civiles, desestructuración indígena y transición al sistema colonial (1537-1369). En Historia de América Andina. Formación y apogeo del sistema colonial (siglos XVI-XVII), ed. Manuel Burga, 71-98. Quito: Universidad Andina Simón Bolívar.

Harner, Michael J. (1978). Shuar Pueblo de las Cascadas Sagradas. Quito: Ediciones Mundo Shuar.

Herrera, Marta (2009). Cultura y guerra. Los Sindagua de la Laguna de Piusbí (el trueno) a comienzos del siglo XVII, Historia Critica 1: 68-79.

Hocquenghem, Anne-Marie (1993). Rutas de entrada del Mullu en el extremo Norte del Perú. Bull. Inst. fr. Études andines. 22, no, 3: 710-719.

Howitt, Robert (1993). A World in a Grain of Sand': Towards a Reconceptualization of Geographical Scale. Australian Geographer, $24 \mathrm{n}^{\circ}$ 1: 33-44.

Hyslop, John (1998). Las fronteras estatales extremas del Tawantinsuyu. En Las fronteras del Estado Inca, comps. Tom Dillehay y Patricia Netherly, 33-51. Quito: Fundación Alexander von Humbolt/ Ediciones Abya-Yala.

Ibarra, Ana Carolina (1994). Las fronteras en América Latina al concluir la lucha por la independencia. En Las fronteras en Iberoamérica: aportaciones para su comprensión histórica, comp. David Piñera, pp. 51-68. México: Universidad Autónoma de Baja California.

Jackson, Robert (2009). Missions on the Frontiers of Spanish America, Journal of Religious History, 33, $n^{\circ} 3: 328-347$.

Johnson, Corey y George White (2011). Traces of Power: Europe's impact on the political organization of the globe. En Engineering Earth, ed. Staley Brunn, 2139-2157. Holanda: Springer. 
Jurado Noboa, Fernando (1990). Esclavitud en la Costa Pacifica: Iscuandé, Barbacoas, Tumaco y Esmeraldas. Siglos XVI al XIX. Quito: Ediciones Abya-Yala.

Kubler, George (1947). The Neo-Inca State (1537-1572). The Hispanic American Historical Review, 27, $n^{\circ} 2: 189-203$.

Laviana, María Luisa (1987). Guayaquil en el siglo XVIII: recursos naturales y desarrollo económico. Sevilla: Escuela de Estudios Hispano-Americanos.

Levaggi, Abelardo (2002). Diplomacia hispano-indígena en las fronteras de América: historia de los tratados entre la monarquía española y las comunidades aborígenes. Madrid: Centro De Estudios Políticos y Constitucionales.

Lozano, Alfredo (1991). Quito, ciudad milenaria: forma y símbolo. Quito: Ediciones Abya Yala.

Lucena, Manuel (1993). Laboratorio Tropical. Caracas: Monte Ávila Latinoamericana.

Lucena, Manuel (1996). El reformismo de frontera. En El reformismo borbónico, ed. Agustín Guimerá, 265-276. Madrid: Alianza Editorial.

Maier, Georg (1969). The boundary dispute between Ecuador and Peru. American Journal of International Law, 63: 28-46.

Maldonado, Luis (2006). Movimiento Indígena y participación política en Ecuador. En Incidencias de participación politica en los pueblos indígenas, ed. Fundación Konrad Adenauer, 25-40. Guatemala: FKA.

Manrique, Luis Esteban G. (2006). De la conquista a la globalización. Estados, naciones y nacionalismos en América Latina. Madrid: Biblioteca Nueva.

Mires, Fernando (2005). La rebelión permanente: las revoluciones sociales en América Latina. México DF: Siglo XXI Editores.

Montoya, Juan David (2011). ¿Conquistar indios o evangelizar almas? Políticas de sometimiento en las provincias de las tierras bajas del Pacífico (1560-1680), Historia Crítica 45: 10-30.

Moreno, Segundo (1976). Sublevaciones indígenas en la Audiencia de Quito, desde comienzos del siglo XVIII hasta finales de la colonia. Bonn: B.A.S.

Muratori, Ana María (2007). Fronteras: conceptos y caracterización espacio-temporal. En Fronteras europeas y latinoamericanas, coord. Lorenzo López, 13-26. León: Universidad de León.

Muratorio, Blanca (1981). Misioneros e indios en la amazonia norte del Ecuador. En Etnicidad, evangelización y protesta en el Ecuador, Blanco Muratorio, 43-68. Quito: CIESE.

O`Donnell, Guillermo (2002). Acerca de las problemáticas fronteras de América Latina contemporánea. En Cruzando fronteras en América Latina, comp. CEDLA, 7-18. Amsterdam: CEDLA.

Oberem, Udo (1980). Los Quijos: historia de la transculturación de un grupo indigena en el oriente ecuatoriano. Otavalo: Instituto Otavaleño de Antropología.

Ospina, Pablo (1992). La región de los Quijos: una tierra despojada de poderes (1578-1608), Procesos: Revista Ecuatoriana de Historia, 3, n 2: 3-31.

Pérez Marcos, Regina María (2002). Aspectos institucionales de la aculturación indígena en la gestación de la sociedad colonial peruana. En Derecho y administración pública en las Indias Hispánicas coord. Feliciano Barrios, pp. 1321-1349. Cuenca: Ediciones de la Universidad de Castilla-La Mancha.

Poma de Ayala, Guaman (1980). Nueva corónica y buen gobierno, Tomo I. Caracas: Biblioteca Ayachucho.

Portais, Michel (1983). De los cazadores recolectores hacia el sistema colonial del dominio del espacio. En El manejo del espacio en el Ecuador-Etapas claves, Jean Paul Deler, Nelson Gómez y Michel Portais, pp. 1-102. Quito: Centro Ecuatoriano de Investigación Geográfica. 
Regalado, Liliana (1997). El inca Titu Cusi Yupanqui y su tiempo. Lima: Fondo Editorial de la Pontificia Universidad Católica del Perú.

Renard-Casevitz, France-Marie, Thierry Saignes y Anne Christine Taylor (1988). Al este de los Andes. Quito: Ediciones Abya Yala.

Rhi-Sausi, José Luis y Nahuel Oddone (2009). Fronteras y cooperación transfronteriza en América Latina: introducción al Proyecto Fronteras Abiertas. En Cooperación Transfronteriza e Integración en América Latina coords. José Luis Rhi-Sausi y Dario Conato, 11-32. Roma: IILA y CeSPI.

Ribeiro, Darcy (1992). Las Américas y la Civilización. Proceso de formación y causas del desarrollo desigual de los pueblos americanos. Caracas: Biblioteca Ayacucho.

Romero, Ximena (2003). Quito en los ojos de los viajeros: el siglo de la ilustración. Quito: Ediciones Abya Yala.

Romoli, Katheen (1963). Apuntes sobre los pueblos autóctonos del litoral colombiano en la época de la conquista española. Revista Colombiana de Antropología 12: 262-290.

Rostworoski, María (1988). Historia del Tawantinsuyu. Lima: Instituto de Estudios Peruanos.

Ruiz Mantilla, Lucy (1992). Jumandi: rebelión, anticolonialismo y mesianismo en el oriente ecuatoriano, Siglo XVI. En Opresión colonial y resistencia indigena en la Alta Amazonia comp. Fernando Santos, pp. 77-102. Quito: Ediciones Abya Yala.

Salomon, Frank (1978). Pochteca and mindalá: a comparison of long-distance traders in Ecuador and Mesoamerica, Journal of the Steward Anthropological Society, 9, no 1-2: 231-246.

Santos, Fernando y Federica Barclay (2002). La frontera domesticada: historia económica y social de Loreto. Lima: Fondo Editorial de la Pontificia Universidad Católica de Perú.

Santos, Fernando (2004). Los Yánesha. En Guía Etnográfica de la Alta Amazonia IV eds. Fernando Santos y Federica Barclay, 159-360. Panamá: Smithsonian Tropical Research Institute; Lima: Instituto Francés de Estudios Andinos.

St. John, Ronald Bruce (1994). The boundary between Ecuador and Peru. Boundary and territory briefing, $1, n^{\circ}$ 4: 1-23.

Tapia, Luis (2004). Territorio, territorialidad y construcción regional amazónica. Quito: Ediciones Abya Yala.

Taylor, Anne Christine (1994). El Oriente ecuatoriano en el siglo XIX: el otro litoral. En Historia y región en el Ecuador: 1830-1930, ed. Juan Maiguashca, 17-67). Quito: Corporación Editora Nacional.

Torres-Londoño, Fernando (2012). Visiones jesuíticas del Amazonas en la Colonia: de la misión como dominio espiritual a la exploración de las riquezas del río vistas como tesoro. Anuario Colombiano de Historia Social y de la Cultura, 39, $\mathrm{n}^{\circ}$ 1: 183-213.

Trujillo, Patricio (2001). Salvajes, civilizados y civilizadores. La Amazonia ecuatoriana: Espacio de las ilusiones. Quito: Ediciones Abya Yala.

Turpo, Aureliano (2006). Estado plurinacional reto del siglo XXI. Camino hacia la Asamblea Constituyente. Propuesta politica kechua tawantinsuyana. La Paz: Plural Editores.

Turpo, Aureliano (2011). La descolonización: hito histórico y politico para la construcción de la sociedad comunitaria plurinacional del siglo XXI. La Paz.

Wade, Peter (2002). Introduction: The Colombian Pacific in Perspective. Journal of Latin American Anthropology. 7, no 2: 2-33.

Yampara, Simón (2005). Descentralización y autonomía desde la visión de los pueblos originarios. Ponencia presentada en Seminario Taller Visiones indígenas de descentralización, febrero 24, en Huajchilla, La Paz, Bolivia.

Zuluaga, Francisco Uriel y Mario Diego Romero (2007). Sociedad, cultura y resistencia negra en Ecuador. Cali: Editorial Universidad del Valle. 